\title{
Why should we monitoring sarcopenia in the middle-aged and older patient?
}

Keywords: sarcopenia, muscle atrophy, muscle strength, functionality, older adults

Abbreviations: $\mathrm{CDC}$, centers of disease control and prevention; ICD-10, international classification of diseases $10^{\text {th }}$ revision; EWGSOP, sarcopenia working group on sarcopenia in older people; ICU-AW, intensive care unit-acquired weakness

\section{Introduction}

From a broad perspective, for a long time, the importance of muscle mass for the organic system was circumscribed with its relationship with body movement. Nevertheless, this organ was not discussed with its due importance, so that the cellular and biological mechanisms responsible for bone maintaining were pointed as the most important. In the course of time, muscle mass began to be associated with an esthetics and beauty, product of muscular adaptations presented by athletes from ancient Greece to modern times. Nowadays, muscle mass is indicated as one of the most essential elements in the organic system, once this demonstrated capacity to act as an active endocrine organ, secreting signaling molecules (i.e., myokines), which may alter the function of several systems, such as endocrine, immune and nervous. Moreover, several researchers have studying the impact of skeletal muscle plasticity in the functioning of our biological system.

Basically, two adaptations can occur in the skeletal muscle. The first one is called as muscle hypertrophy, and is characterized for an increase in the contractile units of sarcomeres (e.g., actin and myosin). This adaptation occurs when the skeletal muscle has been under went to challenges that it cannot totally cope with. To a classic vision of this phenomenon, you must follow someone who is beginning to exercise in a gym. For many reasons that are outside the context of this manuscript, it is necessary to imagine a young and healthy subject who will do resistance training or strength training. In the first weeks of training, just few or no changes will be observed. However, after approximately two months of resistance training, an increased muscle mass may be observed.

The second adaptation is called muscle atrophy. Contrarious to muscle hypertrophy, muscle atrophy is characterized as the loss of muscle mass, caused by significant reduction in the size of the sarcomeres proteins. Muscle atrophy is presented in different conditions, including, but not limited to aging. In fact, few weeks after the limb immobilization due to a bone fracture, for example, is possible observe that the size of the immobilized limb is substantially reduced in comparison with the non-immobilized limb. One of the first medical records about muscle atrophy are dated from the early fifties, when Leonardo da Vinci, after performed his first dissection in an old man, postulated that "muscles were consumed and reduced to the state of a thin membrane so that the cords, instead of being transformed into muscle were converted into a wide sheet".

Due its close association with muscle strength, and, consequently, muscle functionality, muscle mass per se was used by several studies as a synonym of sarcopenia, mainly before the 2000's. In fact, in
Volume I Issue 3 - 2017

Hélio José Coelho Junior

School of Physical Education, University of Campinas, Brazil

\begin{abstract}
Correspondence: Hélio José Coelho Junior, Applied Kinesiology Laboratory LCA, School of Physical Education, University of Campinas, Av. Érico Veríssimo, 70I. Cidade Universitária Zeferino Vaz, Barão Geraldo, CEP |3.083-85I, Campinas SP, Brazil, Email coelhojunior@hotmail.com.br
\end{abstract}

Received: May 19, 2017| Published: August 02, 2017

this time, there was not a consensus about the clinical diagnosis of sarcopenia. However, recently, in 2016, the Centers of Disease Control and Prevention (CDC) established sarcopenia as a pathology (i.e., International Classification of Diseases 10th Revision [ICD10]; M62.84), which is concepted as a condition characterized for decreasing in skeletal muscle mass (i.e., muscle atrophy) concomitantly with a state of muscle weakness, product of impaired muscle strength and/or physical functionality (e.g., walking, sit and reach).

In this sense, operational definitions of sarcopenia using cutoffs have been publishing by different laboratories and research groups around the world. ${ }^{2-4}$ Is important to mention, that previous the CDC statement, sarcopenia was considered a geriatric syndrome and these operational definitions were widely accepted not only in the research field, but also in the clinical practice of physical educators, nutritionists, occupational therapists, physiotherapists and others health professionals, as a profitable tool to characterize sarcopenia in older adults, once there was a not solid construct universally accepted about sarcopenia.

The European Consensus of Sarcopenia was created by the Sarcopenia Working Group on Sarcopenia in older people (EWGSOP) and is, probably, the most accepted operation definition of sarcopenia. ${ }^{2}$ In this document, the researchers postulated that three classes of sarcopenia can be observed in the patients: presarcopenia, sarcopenia and severe sarcopenia. Muscle atrophy is showed in all cases and differences among the levels are explained by the presence or not of dynapenia (i.e., loss of muscle strength) and impaired muscular functionality.

Therefore, a patient with presarcopenia presents marked decrease in muscle mass, without association with dynapenia or decreased muscular functionality. In turn, to be diagnosed as sarcopenic, the patient should show a state of muscle atrophy associated with decreased muscle strength or muscle functionality. Lastly, in the severe sarcopenia, all the aforementioned factors should be presented. ${ }^{2}$

The incidence of sarcopenia rises with aging and its prevalence is markedly increased in older adults. ${ }^{5}$ In these patients, sarcopenia is considered primary, once this occurs in response to the myriad of physiological alterations associated with aging process (e.g., inflammation, oxidative stress). The most concern about sarcopenia is 
due its relationship with the development of low physical functionality and mobility, which prejudices patient's independence, impairing its capacity to perform the activities of daily living (ADL). ${ }^{6}$

Moreover, a growing number of evidence have been demonstrating that sarcopenia is not only associated with poor skeletal muscle functionality, but also with low glucose metabolism, ${ }^{7}$ and vascular alterations, ${ }^{8}$ resulting in a poor prognosis in these individuals. In fact, data from our laboratory, for example, demonstrate that sarcopenia patients showed a 3.1-fold increased risk of having higher values of pulse pressure - a marker of arterial stiffness - in comparison with nonsarcopenic women. ${ }^{8}$

However, is important to mention, that sarcopenia may occur in response to conditions imposed by the environment, as under nutrition, limb immobilization, and Intensive Care Unit-Acquired Weakness [ICU-AW]. ${ }^{9}$ Regarding ICU-AW, this is a typical clinical condition which demonstrates that sarcopenia does not occur only in older adults, but also in middle-aged adults underwent to peculiar situations. ${ }^{9}$ This phenomenon occurs in ICUs, where the patients show symmetrical muscle weakness, which is first developed in the lower-limbs, and a posteriori, affects the upper limbs and, possibly, the respiratory muscles. ${ }^{9}$

Therefore, is important observe that just recently sarcopenia has gained a status of disease and there is a growing number of data in this context. Nevertheless, evidence indicates that monitoring of sarcopenia in middle-aged and older adults can collaborate to avoid a poor prognosis associated with neuromuscular, metabolic and vascular alterations in these patients.

\section{Acknowledgements}

The author thanks the editors of the journal for the invitation to write the manuscript.

\section{Conflict of interest}

The author declares that he has no conflicts of interest.

\section{References}

1. Tonelli F. As Leonardo da Vinci discovered sarcopenia. Clin Cases Miner Bone Metab. 2014;11(1):82-83.

2. Cruz-Jentoft AJ, Baeyens JP, Bauer JM, et al. Sarcopenia: European consensus on definition and diagnosis: report of the european working group on sarcopenia in older people. Age Ageing. 2010;39(4):412-423.

3. Iolascon G, Di Pietro G, Gimigliano F, et al. Physical exercise and sarcopenia in older people: position paper of the Italian society of orthopaedics and medicine (OrtoMed). Clin Cases Miner Bone Metab. 2014;11(3):215-221.

4. Chen LK, Liu LK, Woo J, et al. Sarcopenia in Asia: consensus report of the Asian working group for sarcopenia. $J$ Am Med Dir Assoc. 2014;15(2):95-101.

5. Diz JBM, Leopoldino AAO, Moreira BDS, et al. Prevalence of sarcopenia in older Brazilians: A systematic review and meta-analysis. Geriatr gerontol Int. 2016;17(1):5-16.

6. Morley JE, Abbatecola AM, Argiles JM, et al. Sarcopenia with limited mobility: an international consensus. $J$ Am Med Dir Assoc. 2011;12(6):403-409.

7. Kim KS, Park KS, Kim MJ, et al. Type 2 diabetes is associated with low muscle mass in older adults. Geriatr Gerontol Int. 2014;14:115-121.

8. Coelho Júnior HJ, Aguiar SdS, Gonçalves IdO, et al. Sarcopenia is associated with high pulse pressure in older women. $J$ Aging Res. 2015;109824:1-6.

9. Kizilarslanoglu MC, Kilic MK, Dogrul RT, et al. Sarcopenia and length of hospital stay. Is this a vicious cycle. Eur J Clin Nutr. 2016;70(7):863. 